\title{
Characteristic of Households with Women Using Unskilled Birth Attendants in Urban Slum of Kisumu East District Nyalenda Community Unit
}

\author{
Celestine A. Okang*, Oyugi Henry \\ Tropical Institute of Community Health and Development, Great Lakes University of Kisumu, Kenya
}

Copyright $@ 2017$ by authors, all rights reserved. Authors agree that this article remains permanently open access under the terms of the Creative Commons Attribution License 4.0 International License

\begin{abstract}
Maternal and child mortalities can be reduced if all women deliveries are attended by trained and skilled birth attendants that can provide Emergency obstetric care. Community health strategy has been put in place as an intervention to reduce maternal mortalities. In the urban slum of Kisumu a third (34.7\%) mothers still use unskilled attendant. This study set out to investigate characteristic of households with women using unskilled birth attendants in urban slum of Nyalenda, kisumu, Kenya. The study was comparative in design in which all the households with women who used unskilled attendants during their last delivery included in the study as cases, next nearest, neighboring households with women who used skilled attendants in the last delivery, included as controls, at a ratio of $1: 1$. Therefore, a total of 1033 cases and 1033 controls were analyzed with a total sample of 2066 as valid cases. The study showed age group 35 and above was higher among the mothers using unskilled delivery (14\%) as compared to those using skilled attendants (10.3\%), while mothers who had secondary and above level of education were less likely to have unskilled birth attendant odds ratio (OR) $=0.420$ (95\% $\mathrm{CI}=0.257-0.685)$ as compared to mothers with primary level of education $\mathrm{OR}=0.552(95 \% \mathrm{CI}=0.470-0.649)$. Based on the study results, the odds ratio showed minimal difference on the household characteristic with only individual characteristic showing some significant results on, age, education level and ante natal clinic (ANC). The study found that older mothers do not go for skilled attendance, despite the fact that most young mothers go for skilled attendance, it is a concern that the older mothers who should attend skilled delivery tend not to go yet they are a risk factor in terms of delivery and childbirth, and hence they need to use maternity health care services from skilled health personnel.
\end{abstract}

Keywords Skilled Delivery, Unskilled Delivery, Urban Slums

\section{Introduction}

Maternal mortality, also known as maternal death, continues to be the major cause of death among women of reproductive age in many countries and remains a serious public health issue especially in developing countries (WHO, 2008) ${ }^{[1]}$.

Childbirth is a normal physiological process, which can become pathological due to the adoption of certain practices and consequently affect the health and survival of the mother and newborn. Good pre and postnatal care and trained assistance at the time of childbirth are thus very important to ensure maternal and child survival (S.; Bhattacharya, M. et al 1993) ${ }^{[2]}$. The World Health Organization (WHO) estimated that in 2008 that there were 342,900 maternal deaths relating to pregnancy and childbirth, most of them occurred in developing countries (WHO 2010) ${ }^{[3]}$. Pregnancy and child birth complications are leading causes of death and disability among women of the reproductive age in developing countries. There are practices adopted by unskilled attendants such as delay in referral of excessive post-partum bleeding and unhygienic practices during delivery which can cause serious harm to both the mother and the baby. Thus unskilled attendants have been blamed for being incapable of providing adequate services due to their lower level of literacy and knowledge and because of the harmful practices they adopt during home births (Butlerys et al., 2002 GoI 2000a Guha 2005; 1998, UNICEF 2004, WHO 1998, 1998b) [4].

In the low and middle-income countries many deliveries still occur at home with the assistance of unskilled birth attendant, these deliveries take place even in urban slums having close proximity to the health facility and as a result, it has contributed to the continuing rise in maternal mortality rates affecting the developing countries. Over 50\% of births in slums occur at home, mostly in poor hygienic conditions and under untrained assistance (Sheela Saravanan et al., 2008) ${ }^{[5]}$. 
Sub-Saharan Africa is the only region of the developing world in which the proportion of births attended by skilled health personnel remained essentially stagnant from 1990 to 2005. This indicator increased from $42 \%$ to $45 \%$ in this time period. The continuing substandard maternal health care and delivery situation in developing countries especially in Sub-Saharan Africa has remained a cause of concern for public health researchers and policy makers, the low uptake of maternal services and facility delivery have been topics of research in the field of medicine and sociology (Sheela Saravanan et al., 2008) ${ }^{[5]}$. In sub-Saharan countries, home deliveries are over $60 \%$ taking place largely in rural and urban areas and almost $50 \%$ of women give birth with the assistance of unskilled attendants (WHO 2006) ${ }^{[6]}$. The implication is that every minute, at least a woman dies from pregnancy and childbirth in these regions (WHO, 2005) ${ }^{[7] .}$

Kenya is among the sub-Saharan countries with high maternal mortality ratios, at 488 per 100,000 live births (KDHS08-09) ${ }^{[8]}$. The 2008-09 KDHS found that two out of five births (43 percent) are delivered in a health facility, while 57 percent are delivered outside a health institution or at home with the assistance of unskilled birth attendants. In most rural areas and urban slums, women who deliver at home are assisted by Traditional Birth Attendants (TBA's), family members, friends or neighbors. Worse still, the deliveries are conducted in unhygienic environments predisposing the mothers and babies to postpartum and Neonatal sepsis (Sheela Saravanan et al., 2008) ${ }^{[5]}$. In Kenya, 28 percent of births are assisted by traditional birth attendants, relatives and friends assist by 21 percent of births while 7 percent of mothers do not receive any form of assistance (KDHS08-09) ${ }^{[8]}$.

Kisumu East Health District Office records show that problems related to pregnancy and childbirth resulted in the deaths of 42 women in 2008, 41 women in 2009 and 39 women in 2010. This translates to 456, 434 and 405 maternal deaths per 100,000 live births, respectively. Some of these deaths occurred as a result of complication during delivery which were assisted by unskilled attendant at home and referred late to the hospital for assistance (Kisumu East Health District Office records). These rates are unacceptably high but they are not surprising given that, nationwide, Kenya's maternal mortality ratio increased from $414 / 100,000$ in 2003 to 488/100,000 in 2008 (KDHS, 2003; KDHS, 2008 ${ }^{[8]}$ Kenya.

In peri-urban setting, poor organization and access to maternal health services has always been a major challenge Omo-Aghoja et al. (2008) ${ }^{[9]}$ asserted that maternity care in Kenya is organized around four tiers of care community, primary, secondary and tertiary levels of care. The primary level of care has health centers located in every community unit that is according to community health strategy. Pregnant women are to receive antenatal care, delivery and postnatal care in the primary health centers nearest to them which is not the case in the peri-urban setting of Nyalenda. In case of complications they are referred to secondary care centers, managed by County government, or tertiary centers, managed by the National government.

In the urban slum unskilled birth attendants consider themselves as private practitioners who respond to request for service and receive compensation in return (Singh 1994) [10]. As they receive compensation in cash or in kind from their clients' family, they make their services affordable since they generally accept whatever is offered to them (Jeffery et al., 1984, Singh 1994) ${ }^{[11]}$. Given the cost involved in accessing the formal health care service in Kenya, unskilled attendants are much more affordable to impoverished people (Banerjee, A., Deaton, A. \& Duflo, E. $(2004)^{[12]}$. Researchers have observed that at present there is a serious shortage of professionally trained midwives in developing countries and therefore have cautioned that unskilled birth attendants will continue to exist despite changes in government policies (Kamal 1998) ${ }^{[13]}$. This is much higher than the developing country average of 290/100,000. The implementation of community health strategy in Nyalenda, supported by Great Lakes University of Kisumu, realized minimal improvement in maternal health indicators when compared to the rural and nomadic counterparts (Olayo et al. 2014) ${ }^{[14]}$. About a third (34.7\%) mothers were still using unskilled delivery despite the fact that health facilities are within less than $5 \mathrm{~km}$ radius. This study set out to investigate characteristic of households with women using unskilled birth attendants in urban slum of Nyalenda, kisumu, Kenya.

\section{Methodology}

The study was comparative in design in which all the households with women who used unskilled attendants during their last delivery being included in the study as cases, with the next nearest, neighboring households with women who used skilled attendants during the last delivery, being included as controls, at a ratio of 1:1. The study used the entire cases against controls based on the inclusion criteria. Therefore a total of 1033 cases and a total of 1033 controls were analyzed in the study with a total sample of 2066 as valid cases.

The study used Secondary quantitative data already collected from household register undertaken as part of the implementation of the Community Health Strategy, the establishment of Community Units (CUs). The secondary data bases were checked for accuracy, by re-entering $10 \%$ of household registers and frequencies using Statistical Package for Social Science (SPSS) version 20, using descriptive statistics. Microsoft Excel programme was used for graphical presentation of data. The selection of the 2 community units and 9 sub community units for data collection were based on the sites where Great Lakes University of Kisumu (GLUK) was involved as partnership research sites.

Descriptive analysis was used to describe distribution of the study population by age, marital status, ANC attendance 
and residence. Cross tabulation was carried out comparing cases and controls with regards to background variables, the differences between cases and controls with regards to various factors which was determined using the odds ratio. Pearson Chi-squared analysis and odds ratios were calculated to measure the strength of the relationship between the unskilled birth attendant (dependent variable) and the various predictor variables. All tests were conducted for $95 \%$ confidence interval with $\alpha=0.05$.

Ethical approval was obtained from the ethical review board of Great Lakes University through the department of community health and development. At data collection informed consent was obtained from the respondents before conducting the interview. This was done by explaining to the respondent of their right to participate or withdraw from the study at any time for any reason.

\section{Policy Implication}

The information generated will increase awareness among service providers, managers and policy makers on the barriers that limit access to skilled delivery. Such information could inform policy modification aimed at removing critical barriers limiting access to care at the time of need, influence changes in messages communicated to consumers, as well as practices of service providers and managers that may be contributing to the choice of unskilled service provider as the preferred source of care at the time of delivery.

The findings will inform county government, district team, health managers and the health facility in planning of delivery services programme and to guide community advocacy efforts, inform decision making concerning resource allocation and generation of robust data to provide estimate in the future, targeting the households that are hardest to reach.

It will also be used for future monitoring and evaluation, which will be used for evidence-based dialogue informed by community- and facility-based information systems, complemented by knowledge of barriers that are unique to the hardest to reach households.

\section{Results}

There was an apparent difference in the proportion of age group 25-29 which seemed to be lower (25.8\%) among mothers using unskilled attendants as compared to those using skilled attendants (28.4\%). The age group 35 and above was higher among the mothers using unskilled delivery (14\%) as compared to those using skilled attendants (10.3\%), see table 1.0. Of young mothers aged 15 to 34, $89.6 \%(926 / 1033)$ had skilled birth attendant as compared to $86 \%$ (888/1033) of young mothers who had unskilled birth attendant while women aged 35 to 49 (Old mothers) $10.4 \%$ (107/1033) had skilled birth attendant as compared to $14 \%$ (145/1033) of old mothers who had unskilled birth attendants. It would appear that the age groups 20 to 34 was higher among mothers using skilled attendants and age group 35 and above was higher among mothers using unskilled attendants, see table 1.0. Over all the young mothers were less likely to have unskilled birth attendance with an OR= $0.977(95 \% \mathrm{CI}=0.949-1.007)$ as compared to the old mothers with $\mathrm{OR}=1.165$ (95\% CI= $0.961-1.413)$.

The mother's education was measured by the level of schooling completed: none, primary, secondary and above. The study revealed that out of those maternal mothers who had skilled birth attendant, 1.8\% (19/1033) had no formal education, 54.5\% (563/1033) had primary level of education and $43.7 \%$ (451/1033) had secondary and above level of education. This compared to the cases of mothers who had unskilled birth attendant, 3.1\% (32/1033) had no formal education, 66.9\% (691/1033) had primary education and $30.0 \%$ (310/1033) had secondary and above level of education. Thus the percentage of women who had secondary and above level of education and received unskilled birth attendant gradually decreased to $30.0 \%$.

Mothers who had secondary and above level of education were less likely to have unskilled birth attendant $\mathrm{OR}=0.420$ (95\% CI $=0.257-0.685)$ as compared to mothers with primary level of education $\mathrm{OR}=0.552(95 \% \mathrm{CI}=0.470-$ 0.649).

ANC attendant ( $4+$ times) was included as a variable in the analysis to find out if it was associated with whether a woman delivers at home or not. A large proportion of mothers who had skilled birth attendant 83.1\% (858/1033) had ANC (4+times) as compared to only 35.5\% (367/1033) mothers who had unskilled birth attendant. Women who obtained antenatal care (4+ times) was significantly more likely to deliver at a health facility than those that did not.

Women who had ANC (4+ times) attendance were less likely to have unskilled birth attendance OR=0.291 (95\% $\mathrm{CI}=0.263-0.323)$ as compared to women who had no ANC $(4+$ times $) \mathrm{OR}=2.3(95 \% \mathrm{CI}=2.112-2.505)$ mothers who had ANC (4+ times) attendance were 2 times more likely to have health facility delivery. 


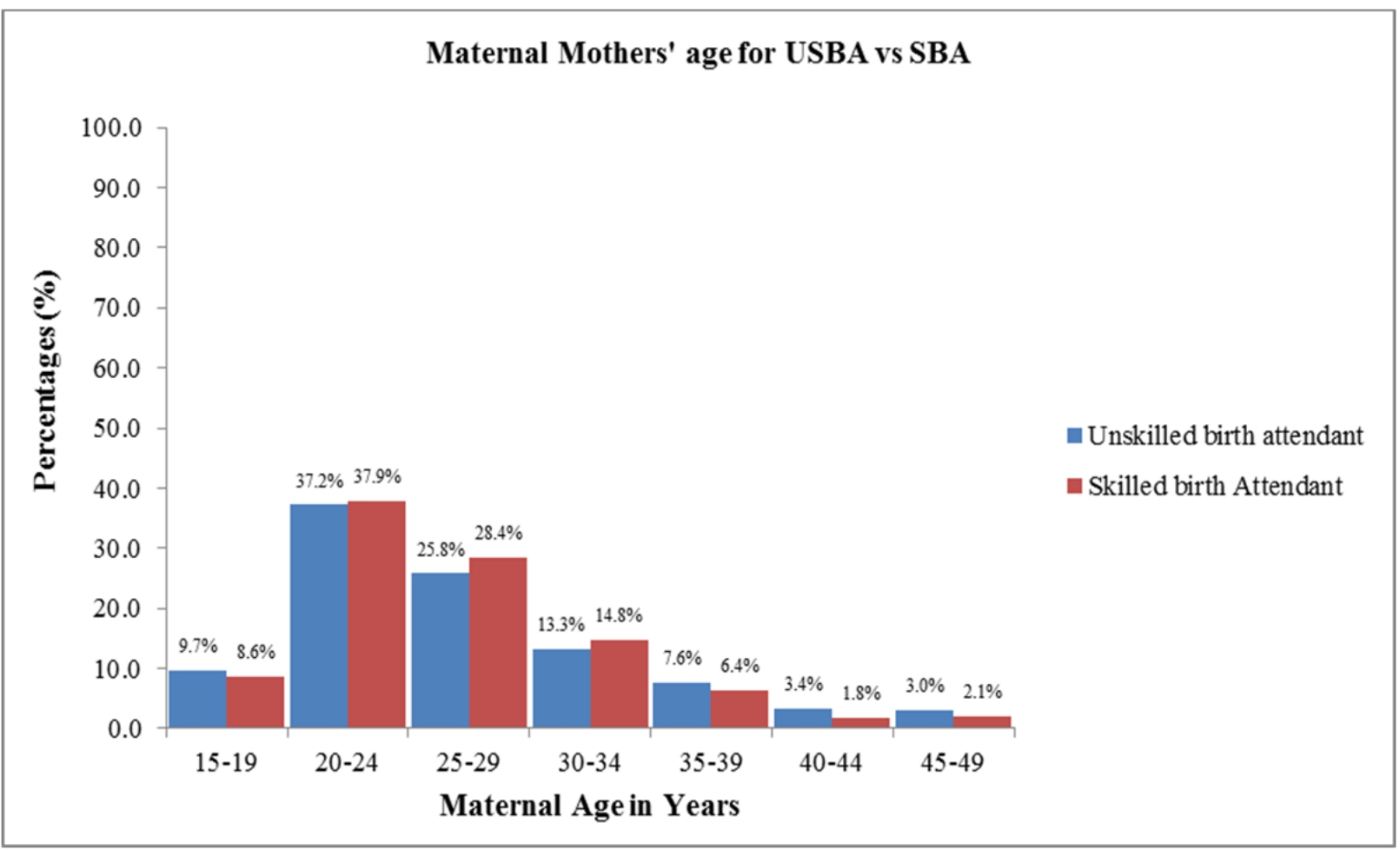

Figure 1. Rate of use of unskilled delivery by maternal age

Table 1. Demographic characteristics of Woman's age distribution by delivery attendant

\section{Characteristics}

Overall

N

189
775
560
290
145
54
53

2066

\section{Unskilled attendant \\ n \\ $\%$}

100

384

267

137

79

35

31
(9.7)

(37.2)

(25.8)

(13.3)

(7.6)

(3.4)

(3.0)
Skilled Attendant

n

$\%$

89

391

293

153

66

19

22

Table 2. Characteristics of Woman's education by delivery attendant

\begin{tabular}{lcccccc}
\hline Characteristics & \multicolumn{2}{c}{ Overall } & \multicolumn{2}{c}{ Unskilled attendant } & \multicolumn{2}{c}{ Skilled Attendant } \\
& $\mathbf{N}$ & $\%$ & $\mathbf{n}$ & $\%$ & $\mathbf{n}$ & $\%$ \\
EducationalLevel & 51 & $(2.5)$ & 32 & $(3.1)$ & 19 & $(1.8)$ \\
None & 1254 & $(60.7)$ & 691 & $(66.9)$ & 563 & $(54.5)$ \\
Primary & 761 & $(36.8)$ & 310 & $(30.0)$ & 451 & $(43.7)$ \\
Secondaryt & & & & & &
\end{tabular}


Table 3. Socio-economic characteristics of Ante natal care by delivery attendant

\begin{tabular}{lcccccc}
\hline Characteristics & \multicolumn{2}{c}{ Overall } & \multicolumn{2}{c}{ Unskilled attendant } & \multicolumn{2}{c}{ Skilled Attendant } \\
& $\mathbf{N}$ & $\%$ & n & $\%$ & n & $\%$ \\
ANC (4 + times) Attendant & & & & & & \\
Yes & 1225 & $(59.3)$ & 367 & $(35.5)$ & 858 & $(83.1)$ \\
No & 841 & $(40.7)$ & 666 & $(64.5)$ & 175 & $(16.9)$
\end{tabular}

\section{Discussion}

Demographic characteristics of households associated with unskilled delivery by maternal age

In this study it was found that younger mothers, particularly age groups 20 to 34 were significantly more likely to use skilled delivery as compared to the older age groups. This is contradicted by the results of a similar study in Uganda, in which the findings show there is no relationship between maternal age and choice of delivery assistance.

\section{Characteristics of Households Associated with Unskilled Delivery}

The finding of this study suggests that different factors can identify women likely to use unskilled attendant during delivery among slum dwelling women. For example, women's education as predisposing factor had significant impact on delivery service utilization. This agrees with other studies that have shown that maternal education is the most important determinants for health services use (Ensor and Cooper 2004) ${ }^{[15]}$. They argued that better educated women were more aware of health problems, know more about the availability of health care services, and use this information more effectively to maintain or achieve good health status.

Various studies noted that more educated women were more likely to use skilled birth attendants than not educated women who prefer the use of unskilled attendant. A study in Bangladesh found that $74 \%$ of women with more than ten years of education used skilled birth attendants during delivery compared to $18 \%$ who are un educated who used unskilled attendants (Anwar et al. 2007) ${ }^{[16]}$. Yanagisawa in Rural Cambodia also found the same where by women who at least seven years of school attendance being six times more likely to deliver babies at health facility than those who did not attend school (Yanagisawa 2006) ${ }^{[17]}$.

In this study women with secondary and above education delivered in health facilities compared with $3.1 \%$ of the women who had no formal education and used unskilled attendants. Further findings show that Mother who had secondary \& above level of education were less likely to have unskilled birth attendant as compared to mothers with primary level of education.

The findings were consistency with other studies done in different areas, study done by Mrisho in Southern Tanzania showed that mothers with primary and secondary and above level of education were more likely to deliver in health facilities compared to uneducated mothers, also Lwelamira (2012) found that women with higher education in Bahi district they tend to deliver in health facility compared with those with primary or formal education (Mrisho et al. 2007) [18]

This is explained by the fact that educated mothers have more confidence in handling the officials and have the ability and willingness to travel outside the home to seek services. However, contrary findings were also found in delivery assistant in Bangladesh and Thailand.

This may be the fact that education is likely to enhance health knowledge and reduce the power difference between skilled providers and clients so that women developed greater capacity to make decision about their own health needs. Most maternal and child health studies conducted in developing countries strongly agree with this finding (Haque 2009, Babalola and Fatusi 2009) ${ }^{[19]}$.

Socio-economic characteristic of households associated with Antenatal care attendance

Antenatal care (ANC) services provide opportunities for health workers to promote a specific place of deliver or give women information on the status of their pregnancy, which in turn informs their decisions on where to deliver. Risk assessment during ANC may explicitly recommend a place of delivery, for instance to deliver in a hospital for a twin pregnancy. Under normal circumstances, WHO recommends that pregnant mother without any complications have at least four antenatal care clinics to provide sufficient information of her own health and developing foetus. Early booking of antenatal care is important as its provides health worker with the opportunity of early detection of maternal problem and corrective measure taken immediately to get rid of them for benefit of mother and fetus. In this study 83.1\% (858/1033) had ANC (4+times, this proportion is slightly lower than that of national estimate of $92 \%$ (KDHS 2008-2009) ${ }^{[8]}$.

The study also found that $83.1 \%$ (858/1033) of women who attended antenatal clinic 4 plus delivered in health facilities while $35.5 \%$ (367/1033) of those who did not attend antenatal clinic 4 plus delivered at home, although percentage of delivery in health facility in this study is higher than that of national level of $44 \%$ (KDHS 08-09) ${ }^{[8]}$, the relationship between delivery assistant and frequency of antenatal visit was strongly significant as those who attended 
antenatal clinic at least four visit were two times more likely to deliver in health facility compared to those who did not attend antenatal clinic.

Women who had ANC (4+ times) attendance were less likely to have unskilled birth attendance as compared to women who had no ANC (4+ times) i.e. mothers who had ANC (4+ times) attendance were 2 times more likely to have health facility delivery. Yanagisawa et al. (2006) ${ }^{[17]}$, in Cambodia found that antenatal care was a positive determinant of facility delivery only for women who attended the service four times or more (Yanagisawa et al. $2006)^{[17]}$. This might be due to the fact that during antenatal clinic visits, especially if started early, women are provided with health education and information about the benefits of delivering in health facility.

Home delivery and use of unskilled attendant was 35.5\% (367/1033) which is not very high compared to study conducted in Ethiopia where by $76.4 \%$ of the deliveries were assisted by unskilled birth attendants or by relative (Nigussie et al. 2004) ${ }^{[20]}$. Similarly findings had been reported by the study done in rural northern part of Tanzania in which more than ninety percent of women attended antennal clinic but less than half of them delivered in health facility (Magoma et al. 2010) ${ }^{[21]}$.

\section{Conclusions}

Based on the results of this study, there was minimal difference on the household characteristic with only individual characteristic showing some significant results on, age, education level, and ANC. It is still a challenge in urban slum where the study has found that older mothers don't go for skilled attendance, despite the fact that most young mothers go for skilled attendance, it is a concern that the older mothers who should be going for skilled delivery tend not to go and yet they are a risk factor in terms of delivery and childbirth, and hence they need to use maternity health care services from skilled health personnel.

\section{Recommendations}

This study draws the recommendations for both health care management systems researchers and policy makers in order to increase access on skilled delivery and emphasis to the older mothers who still prefer the services of unskilled attendants. For researchers: Further research should be conducted on behavior change on older mothers who use skilled delivery to reach out and convince the older mothers who go for unskilled attendant.

\section{REFERENCES}

[1] World Health Organization (2008), Factsheet, Maternal Mortality, Department of Making Pregnancy Safer.

[2] S.; Bhattacharya, M. et al. 1993

[3] World Health Organization, 2010: working with individual, families and communities to improve maternal and new born health. Geneva: World Health Organization.

[4] Butlerys et al., 2002 GoI 2000a Guha 2005; 1998, UNICEF 2004, WHO 1998, 1998b

[5] Sheela Saravanan et al., 2008 training of traditional birth attendant, an examination of the influence of biomedical frameworks of knowledge on local birthing practices in India.

[6] World Health Organization, 2006: Making difference in countries: Strategic Approach to Improving Maternal and New born Survival and Health.

[7] World Health Organization, 2005: World Health Report 2005: Make every Mother and Child count. Geneva: World Health Organization.

[8] KDHS (2008-2009) Kenya demographic health survey

[9] Omo-Aghoja, L.O., Aisien, O.A., Akuse, J.T., Bergstrom, S., Okonofua, F.E(2010) Maternal Mortality and emergency obstetric care in Benin City, South-south Nigeria Journal of Clinical Medicine and Research Vol. 2, No. 4, pp. 55-60

[10] Singh 1994, Journal of Nurse-Midwifery, 39 (2), PP. 119-123

[11] Jeffery, R., Jeffery, P. \& Lyon, A. (1984) only cord-cutter and childbirth in rural North India, social action, 34(3) pp. 229-250

[12] Banerjee, A., Deaton, A. \& Duflo, E. (2004) Health Care Delivery in Rural Rajasthan Economic and Political weekly, (February 28), PP. 944-949.

[13] Kamal 1998 Kamal, S.M.M., 2009. Factors affecting utilization of skilled maternity care services among married adolescents in Bangladesh. Asian Popul. Stud., 5(2): 153-170.

[14] Olayo et al. 2014

[15] Ensor \& Cooper, (2004), Overcoming Barriers to Health Service Access: Influencing the Demand Side. 15

[16] Anwar et al 2007, Agarwal et al Maternal health care utilization among women in an urban slum in Delhi. Ind. Jr. of comm. Med. Vol. 32, no. 3, July- Sept. 2007 pp. 203-205.

[17] Yanagisawa S, Oum S, Wakai S: Determinants of skilled birth attendance in rural Cambodi. Trop Med Int Health 2006, 11(2): pp 238-251.

[18] Mrisho et al., 2007, Factors affecting home delivery in rural Tanzania. Trop Med Int Health, 12 (7): pp. 862-872.

[19] Haque 2009, Babalola and Fatusi 2009

[20] Nigussie et al. 2004

[21] Magoma et al. 2010 